\title{
Analysis of Representations for Domain Adaptation
}

by Shai Ben-David, John Blitzer, Koby Crammer, Fernando Pereira

presented by Marina Sokolova

\author{
Best-of-NIPS'2006
}




\section{Introduction}

- Domain is a distribution $\mathcal{D}$ on an instance set $\mathcal{X}$

- Domain adaptation of a classifier

A classification task

Source domain $\left(\mathcal{D}_{\mathcal{S}}\right)$

Target domain $\left(\mathcal{D}_{\mathcal{T}}\right)$

Different distributions

Labelled data in source domain

Unlabelled data in both domains

- Examples: spam filters, parsing, part-of-speech tagging 


\section{Suggested approach}

- Motivation: discriminative classification methods are based on the assumption of the same distribution for training and testing data. However, this is not always true.

- Intuition: common representation can make two domains appear similar and enable effective domain adaptation.

- Formalization: a bound on the target generalization error of a classifier trained from labelled data in the source domain. 


\section{Problem Setup}

- $\mathcal{X}$ is the instance set, $\{0,1\}$ is the label set, $\mathcal{Z}$ is a feature set, e.g. $\mathbb{R}^{d}$;

- distribution $\mathcal{D}$ over $\mathcal{X}$, a target function $f: \mathcal{X} \longrightarrow[0,1]-$ common for both domains ;

- a representation function $\mathcal{R}: \mathcal{X} \longrightarrow \mathcal{Z}$ and hypothesis class $\mathcal{H} \subseteq\{g: \mathcal{Z} \longrightarrow\{0,1\}\}$

- $\mathcal{R}$ induces distribution $\widetilde{\mathcal{D}}$ over $\mathcal{Z}$ and its subsets and function $\tilde{f}: \mathcal{Z} \longrightarrow[0,1]:$

$$
\begin{aligned}
& \operatorname{Pr}_{\widetilde{\mathcal{D}}}[B]=\operatorname{Pr}_{\mathcal{D}}\left[\mathcal{R}^{-1}(B)\right] \\
& \tilde{f}(z)=\mathrm{E}_{\mathcal{D}}[f(x) \mid \mathcal{R}(x)=z]
\end{aligned}
$$

- For a predictor $h: \mathcal{Z} \longrightarrow[0,1]$ the expected error is

$$
\epsilon_{\mathcal{T}}(h)=\mathrm{E}_{z \sim \widetilde{\mathcal{D}}_{\mathcal{T}}}|\tilde{f}(z)-h(z)|
$$




\section{Assumptions}

- There is a hypothesis $h \in \mathcal{H}$ that performs well on both domains, i.e. there is exists $h$ and a small $\lambda$ such that $\inf _{h \in \mathcal{H}}\left[\epsilon_{\mathcal{S}}(h)+\epsilon_{\mathcal{T}}(h)\right] \leq \lambda$

- $\mathcal{H}$ has bounded capacity, e.g. VC-dimension $d$. 


\section{Generalization Bound}

\section{Theorem}

If a random labeled sample of size $m$ is generated by applying $\mathcal{R}$ to a $\mathcal{D}_{\mathcal{S}}$ - i.i.d. sample labeled according to $f$, and $\widetilde{\mathcal{U}}_{\mathcal{S}}, \widetilde{\mathcal{U}}_{\mathcal{T}}$ are unlabeled samples of size $m^{\prime}$ each, drawn from $\widetilde{\mathcal{D}}_{\mathcal{S}}$ and $\widetilde{\mathcal{D}}_{\mathcal{T}}$ respectively, then with probability at least $1-\delta$ (over the choice of the samples), for every $h \in \mathcal{H}$ :

$\epsilon_{\mathcal{T}}(h) \leq$

$\hat{\epsilon}_{\mathcal{S}}(h)+\frac{4}{m} \sqrt{d \log \frac{2 e m}{d}+\log \frac{4}{\delta}}+\lambda+d_{\mathcal{H}}\left(\widetilde{\mathcal{U}}_{\mathcal{S}}, \widetilde{\mathcal{U}}_{\mathcal{T}}\right)+4 \sqrt{\frac{d \log \left(2 m^{\prime}\right)+\log \frac{4}{\delta}}{m^{\prime}}}$ A good representation $\mathcal{R}$ achieves low values for training error and domain distance simaltaneously. 


\section{Computing the distance}

- Distance between distributions $\mathcal{D}, \mathcal{D}^{\prime}$ is defined as $d_{\mathcal{A}}\left(\mathcal{D}, \mathcal{D}^{\prime}\right)=2 \sup _{A \in \mathcal{A}}\left|\operatorname{Pr}_{\mathcal{D}}[A]-\operatorname{Pr}_{\mathcal{D}^{\prime}}[A]\right|$,

- Domain distance is to be measure only with respect to function in the hypothesis class where $d_{\mathcal{H}}(.,$.$) indicates the$ distance on the class of subsets $\mathcal{Z}_{h}=\{z \in \mathcal{Z}: h(z)=1\}$.

- A proxy for $d_{\mathcal{H}}\left(\widetilde{\mathcal{U}}_{\mathcal{S}}, \widetilde{\mathcal{U}}_{\mathcal{T}}\right)$ is obtained by training a classifier to discriminate between points generated by source and target distributions. 


\section{Adapting a part-of-speech tagger from the financial to biomedical domains}

- Procedure:

- choose a representation $\mathcal{R}$;

- train a linear classifier using $\mathcal{R}$;

- measure both relevant terms of the bound.

- Building $\mathcal{R}$ (by Structural Correspondence Learning):

- find domain-independent "pivot" features occuring frequently in the unlabelled data in both domains, e.g., determiners, < the token on the left $>$;

- represent other features using their relative co-occurence counts with the pivot features;

- compute a low-dimensional approximation to the co-occurrence matrix (through the singular value decomposition of the matrix).

- Intuition: features from source and target domains which behave similarly for PoS tagging will be represented similarly in the projected space. 


\section{Empirical Results}

- Source : articles from Wall Street Journal (WSJ)

- Labelled: 100 part-of-speech tagged sentences from WSJ

- Target data: biomedical abstracts (MEDLINE)

- Unlabelled data: 500,000 words from each domain

- instances are high-dimensional, binary vectors; $\mathcal{Z}$ is $\mathbb{R}^{d}$, $d=200$

(a) Plot of SCL representation for financial (squares) vs. biomedical (circles)

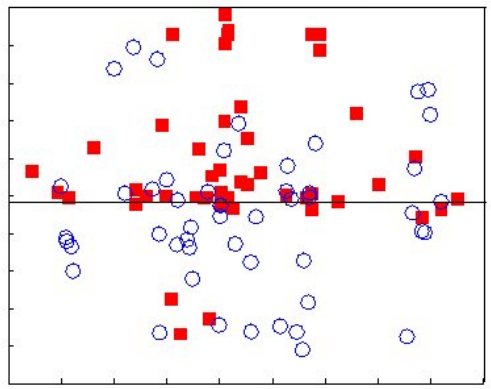

(b) Plot of SCL representation for nouns (diamonds) vs. verbs (triangles)

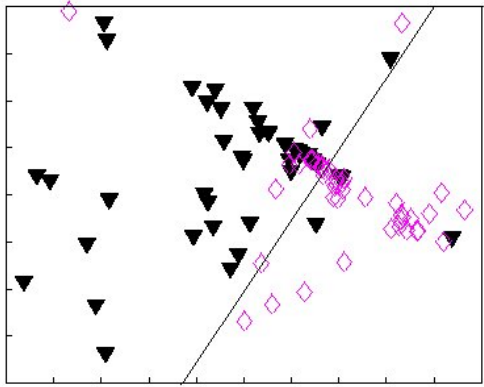




\section{Contributions}

Analysis of a classification problem when a source domain and a target domain have different distributions An upper bound on the generalization of a classifier trained on a source domain and applied on a target domain

\section{Important References}

"Detecting Change in Data Streams", by D. Kifer, S. Ben-David, J. Gehrke, Proc of the 30th VLDB Conference, 2004.

"Domain Adaptation with Structural Correspondence Learning", by J. Blitzer, R. McDonald, F. Pereira, Proc of EMNLP, 2006. 